\title{
What do MRI radiographers really know?
}

\author{
Catherine Westbrook, John Talbot \\ Anglia Ruskin University, \\ Allied Health, \\ East Road, \\ Cambridge \\ CB1 1PT, \\ United Kingdom
}

\section{Keywords \\ MRI; \\ Radiography; \\ Education; \\ Assessment; \\ MRI safety}

\begin{abstract}
Magnetic Resonance Imaging (MRI) is an important diagnostic imaging tool that has seen rapid expansion in recent years with a commensurate increase in the number of radiographers undertaking $\mathrm{MRI}$ scans. This imaging modality is a complex one and the strong magnetic fields associated with MRI scanners pose a very serious risk to patients and staff. Therefore it is essential that MR radiographers receive appropriate training in the correct operation and safe use of MRI. The education of radiographers undertaking MRI examinations is largely provided "in-house" by radiographers or applications specialists. This training is usually informal and focuses on essential safety training and the use of scanner software. This learning is not usually formally assessed and therefore its value is not properly evaluated. In 2007 the authors, acting as independent consultants, developed a number of assessment tools to enable quick and effective evaluation of theoretical knowledge and skills related to the clinical use of $\mathrm{MRI}$ in a group of individuals with a range of $\mathrm{MRI}$ experience. A total of 47 individuals with a range of MRI experience were tested using an Objective Structured Clinical Examination (OSCE). Some were also given a viva voce. Our results show that the majority of radiographers in our sample could not answer more than half the OSCE questions correctly and a significant and unacceptable number did not convince us they could practice MRI safely. Very few of the examinees had adequate knowledge of image quality issues and parameter manipulation. A few also raised concerns over their radiographic credentials.
\end{abstract}

\section{Introduction}

Magnetic Resonance Imaging (MRI) is an important diagnostic imaging tool that has seen rapid expansion in recent years with a commensurate increase in the number of radiographers undertaking $\mathrm{MRI}$ scans. This imaging modality is a complex one, both technically and clinically. In addition, although MRI does not involve the use of ionising radiation, the strong magnetic fields associated with $M R I$ scanners pose a very serious risk to patients and staff. Therefore it is essential that all MR practitioners, especially radiographers, receive appropriate training in the correct operation and safe use of MRI.

Radiographers new to MRI acquire knowledge and skills from other radiographers and applications specialists and through independent learning. Some also attend short courses and participate in post-graduate degree programmes. Except for post-graduate courses, most learning is not formally assessed and therefore its value is not properly evaluated. This means that the knowledge gained by radiographers during training is not established and therefore their ability to practice MRI safely and with understanding can only be assumed.

In 2007 the authors, acting as independent consultants, developed a number of assessment tools to enable quick and effective evaluation of theoretical knowledge and skills related to the clinical use of $\mathrm{MRI}$ in a group of individuals with a range of $\mathrm{MRI}$ experience. The main assessment tool was a Objective Structured Clinical Examination (OSCE). A total of 47 individuals with a range of $\mathrm{MRI}$ experience who gained their radiographic and $\mathrm{MRI}$ training in the UK and overseas were tested using this method. Some were also given a structured viva 
voce. This paper outlines the rationale behind this research, the development of these assessment tools and the results obtained from this assessment. A discussion of our findings and their implications is also included.

\section{Rationale}

In the UK there is very little provision of MRI education at under-graduate level. Currently 25 Universities in the UK deliver BSc Radiography courses but the focus of this education is general radiography. Many BSc Radiography programmes include MRI only as part of a specialist imaging module studied towards the end of the course. Typically this involves some basic theoretical teaching and clinical observation [6]. There are a few dedicated postgraduate MRI courses but relatively few radiographers undertake this level of study. In the UK currently eight Universities offer some form of post-graduate MRI education [7] but there are no published figures of the number of students who participate in this level of education. Based on UK enrolment numbers on the MSc MRI course at Anglia Ruskin University replicated in the other seven Universities, and estimates of the total number of MRI radiographers in the UK, we believe that only $2 \mathrm{e} 3 \%$ of radiographers engage in formal postgraduate education.

There are a few short courses in the UK that focus on essential theoretical topics related to practice which include basic principles, image contrast, image quality, image production, safety and pulse sequences however, like post-graduate courses, there are no published figures for the number of radiographers who attend. As independent consultants, the authors run a short course on MRI theory several times a year in the UK and, based on our attendance figures replicated across other such courses in the UK, we estimate that approximately $15 \%$ of radiographers participate in theoretical MRI short courses every year.

Therefore it appears that most radiographers rely mainly on "in-house" teaching from other radiographers and applications specialists. Although this training is often of high quality, in our experience it is usually focused on MRI safety and the use of scanner software rather than on an in-depth understanding of theoretical MRI concepts. In addition there is usually limited formal assessment of this learning. Assessment is an integral part of the learning process. Unless students are assessed it is impossible for teachers to effectively judge whether the teaching they have delivered has resulted in effective learning and whether gaps in understanding still exist. In educational circles, much emphasis is placed on constructive alignment [3] whereby teaching is implemented in such a way that the learning activities and assessment tasks are aligned with the learning outcomes of the training. Assessment tools are developed with the learning outcomes in mind [2]. This makes the links between learning and assessment transparent and encourages deep learning. Deep learning promotes understanding and application to practice and is accepted as the preferred type of learning as it enables students to solve problems, especially in unusual contexts and to retain this knowledge [18].

Academics in Higher Education endeavour to deliver teaching and assessment tools using these concepts. There are many types of assessment used and each has advantages and disadvantages. The more traditional ones such as examinations and written essays are gradually being replaced with those that reflect the constructive alignment theory and are directly related to the student experience or workplace [3]. However, "in-house" teaching in $\mathrm{MRI}$ is sometimes provided by medical practitioners who are not usually qualified teachers. Therefore teaching may be delivered without any clear learning outcomes and with limited assessment that may not use well constructed educational paradigms.

We wished to test whether there was a need for a simple, reliable assessment tool to evaluate the learning attained by MRI radiographers after both their initial training period and after short courses. This assessment tool must be clearly related to clinical practice to test safety and competency and be as valid and reliable as possible. The aims of this research are to; 
- To assess the level of knowledge related to clinical competence of a group MRI radiographers using an Objective Structured Clinical Examination (OSCE) and a viva voce.

- To make recommendations on how to ensure safe and competent practice of radiographers undertaking $\mathrm{MRI}$ examinations via assessment

\section{Objective Structured Clinical Examination (OSCE)}

There is a substantial body of literature relating to assessment especially in the healthcare professions. A good assessment has both validity and reliability in that it measures outcomes directly related to the student experience and that it consistently achieves the same results with similar types of students [9]. In practice, an assessment is rarely totally valid or reliable as there may be many variables such as ambiguous questions, more than one answer to a question and different opinions or ideas on the part of the assessors [13]. One of the objectives of our research was to develop an assessment tool that could be easily administered to a large group of individuals at one time and could be graded objectively. We also wanted to be able to test a broad range of clinical competencies related to MRI in individuals with a variety of clinical experience. The OSCE assessment tool was selected as it met all of these criteria. An OSCE is a versatile multipurpose evaluative tool commonly used in healthcare education to test skills such as communication, clinical examination, medical procedures and interpretation of results. It is a highly viable and valid clinical examination that provides unique information about the performance of individuals based on objective testing through direct observation [19]. Objective assessment is a form of questioning which has a single correct answer. This removes subjectivity from the assessment and therefore increases its reliability [16].

Typically the OSCE is comprised of several "stations" in which examinees are expected to perform a variety of tasks related to clinical practice within a specified time period. These tasks are formulated against learning outcomes related to particular clinical skills, thus demonstrating competency in these skills [10]. Stations can involve a variety of testing methods, including multiple choice or essay tests, and in the radiography/radiology setting, the use of images. Many studies have shown positive results using the OSCE for evaluating those areas most critical to performance of healthcare professionals, such as the ability to obtain or interpret data, problem-solve, communicate, and handle unpredictable patient behaviors [13]. They are also invaluable for identifying gaps in learning so that additional training can be implemented. Other advantages of the OSCE include the ability to assess a large number of clinically related skills in a relatively short period of time and to establish a standardized evaluation tool using controlled grading criteria related to desired learning outcomes [8].

\section{Viva voce}

The vive voce is an oral examination that is traditionally used by students to defend a piece of work such as a thesis [21]. It helps to establish that the work is that of the students and has not been plagiarized. In this context it was used to elicit patient related skills and knowledge of MRI safety, to extract more advanced level knowledge in certain individuals and to establish the radiographic credentials of some of the examinees.

\section{Methodology}

Forty-seven individuals were assessed using the same OSCE using Microsoft Excel over a period of four months. Thirty-one of these individuals (Sample A) were assessed as part of a wide recruitment process by a private healthcare provider and were trained in MRI overseas. Institute approval was acquired to objectively assess all of these individuals and compare their actual ability to competencies identified through the National Occupational Standards. We were also required to viva voce some of these individuals in Sample A, to weed out unsafe practitioners and provide evidence of our findings. A further 16 individuals (Sample B) were also assessed. They were due to attend an MRI course run by another private healthcare provider and were all trained in MRI in the UK. Sample B was assessed under the same conditions as Sample A and was included in this study so that comparisons could be 
made between the OSCE scores of UK trained and non-UK trained individuals. As the private healthcare provider running the course for Sample B did not require us to perform viva voce examinations, individuals in Sample B did not receive this assessment. Therefore only comparisons in the OSCE scores between the 2 groups have been made.

The test areas in the OSCE were selected as being those most appropriate to safe MR clinical practice and were largely based on the American Registry of Radiologic Technologist (ARRT) content specification for their registry examination in MRI $[1,14]$ which is a timed multiple-choice computer generated examination similar to an OSCE and includes a wide range of theoretical MRI topics applicable to practice. The topics included were;

1. MRI safety and medical terminology (subdivided into 2 sections).

2. Basic principles of MRI.

3. Pulse sequences and image contrast.

4. Image optimisation.

5. Anatomy and pathology (subdivided into 2 sections).

Questions were selected to test a range of knowledge in both basic and more advanced topics related to practice and to assess ability to operate an MRI system safely and with understanding. In particular some questions in the safety section related to the contraindications to MRI and the types of patients who must not be scanned. The section on image optimisation related to recognising common artefacts that an MRI radiographer may expect to see in everyday practice and how to manipulate parameters to optimise image quality. Some questions were multiple choice and in some, the student had to provide a short written answer. Many questions involved the use of MR images and diagrams.

Before performing this test on Samples A and B, a small pilot study was performed on 3 individuals, unrelated to the main study, to test the validity and efficacy of the OSCE. Two individuals were MRI radiographers with a number of years experience, one in clinical MRI and the other as an applications specialist. We used these individuals to test whether the questions could be answered in the allocated time frame and were appropriate for the knowledge we were trying to test, i.e. knowledge applied to clinical practice. The third individual was a non-medical person with no previous exposure to MRI. This individual was selected to ascertain the number of answers that could be correctly answered by guessing alone. The MRI radiographers scored $72 \%$ and $75 \%$ which correlates with expected ARRT examinations scores [1] and appeared to validate the level, range and quantity of questions asked. The non-medical individual scored $16 \%$ by guessing some of the multiple-choice answers correctly, particularly in medical terminology. This was a slightly higher score than we expected based on research in multiple-choice testing methods [4,15] that provide estimates of the chances of guessing a correct answer. In response to this we increased the number of possible answers in the medical terminology sections from 4 to 8 as recommended by this research.

Of the 47 individuals assessed using the OSCE, 16 gained their radiographic qualification in the UK (Sample B) and 31 overseas (Sample A). Those who trained overseas came from India and Sri lanka (12), Nigeria (6), Philippines (6), mainland Europe (3), Zimbabwe (2), South Africa (1) and the Middle East (1). All examinees in both samples had either a Bachelor of Science degree or Diploma in Radiography and all were registered with the Health Professions Council (HPC). The MRI experience of these individuals ranged from 6 months to 15 years with $17(36 \%)$ having three years or more experience in clinical MRI. All of those who had obtained their radiographic qualification overseas had also obtained their MRI training abroad. Those who were UK qualified had also received their MRI training in the UK. None of the examinees had attained a post-graduate qualification in MRI but several had attended short courses in their country of origin. The OSCE was implemented without the examinees, previous knowledge of the test so they did not have prior warning that they were to be assessed and were not therefore able to revise. This was deliberate as we wished to assess each individual's "working" knowledge of MRI and therefore did not want our results skewed by revision prior to the OSCE. 
The examinees were each provided with a laptop with the OSCE preloaded onto the system. Some of the examinees were asked to provide their names; others were anonymous. Those who provided their names were from Sample A and were required to do so by their employers as part of a wider assessment process. The invigilator explained the purpose of the OSCE and how it was to be implemented. One hundred questions, in five subject areas were developed and presented via a Microsoft Excel spreadsheet. Rather than to have separate stations that involves movement of examinees from one computer to the next, each individual sat at one computer that had five separate sections identified as different coloured tabs at the bottom of the screen. Two of these sections were subdivided to test individuals on seven different topics. The examinee was given $10 \mathrm{~min}$ to complete each of the five sections. The OSCE was monitored by an invigilator who instructed the examinees when to move onto the next section. This meant that every individual spent exactly the same amount of time (10 min) on each section.

Thirty-nine examinees in Samples A and B were asked to identify a line drawing of a rotatinganode X-ray tube. This question was added to the end of the OSCE to try to establish the radiographic credentials of the examinees. This was implemented due to some very poor results in the first eight tests performed in Sample A that raised suspicions about the radiographic qualifications of these individuals. Although the OSCE was not primarily aimed at establishing general radiographic credentials, the private healthcare company who had recruited Sample A required that the MRI radiographers they employed perform examinations that involved using ionizing radiation on a rotational basis. Therefore it was necessary to ensure their radiographic credentials were sound. An X-ray tube was selected as it was regarded as an iconic image that all qualified radiographers, irrespective of the qualification achieved, should be able to recognise. This question was therefore added to the end of the OSCE for the remaining 23 examinees in Sample A and all 16 examinees in Sample B. Although it was not necessary to establish the radiographic credentials of Sample B, we wanted to make comparisons between the responses to this question for UK trained vs nonUK trained individuals. The total time for the OSCE was $60 \mathrm{~min}$ which included the initial instruction by the invigilator, completion of each of the five $10 \mathrm{~min}$ sections and the identification of an unlabelled diagram of an X-ray tube.

Each OSCE was automatically marked by the Microsoft Excel software by recognizing the only correct answer to each question. This was achieved by using a formula that recognized a particular word or words and allocated 1 mark if that word was present in the answer box and a zero mark if it was not present. Where the student had to write in the answer, the examiners marked these answers, then checked the accuracy of the automatic marking system and allocated a final mark.

The results of the OSCE examination were scrutinized and some individuals in Sample A were further assessed using a viva voce examination. Those recalled in Sample A for the viva voce were required to do so by their employers as part of a wider assessment process as previously outlined. We endeavoured to viva all examinees in Sample A however due to other commitments of examinees in the induction process of the private healthcare provider, not everyone was able to attend. Despite this 23 of the 31 examinees in Sample A were recalled for a viva voce examination. Examinees achieving marks below $50 \%$ in the OSCE were given a viva voce on patient care related issues, whereas those with marks above $50 \%$ were given a viva voce on advanced MRI topics. All examinees recalled for a viva voce were also given questions to assess their radiographic credentials. This consisted of showing the examinee a photograph of a patient having a lateral cervical spine Xray examination. They were asked to identify the examination taking place and to identify the focus to film distance, the object to film distance and the air gap. They were also asked to explain why an air gap is used. Finally they were asked to identify the collimator and state what it was used for. This examination was selected as it is a common radiographic examination that any qualified radiographer performs routinely. The viva voce examination was delivered by two assessors (the researchers). Each was allocated certain questions to ask the examinee and was equally distributed amongst both assessors. The answers provided by the examinees were recorded by the assessors and mapped against answers considered accurate and appropriate by the assessors. The Sample B population was not given a viva voce examination as this was not required as part of a recruitment process by the private healthcare company involved. 


\section{Results}

The results of the OSCE and viva voce examinations are shown in Figs. $1 \mathrm{e} 8$ and include data from both sample populations. The range of total OSCE scores was $14 \mathrm{e} 72 \%$ with an average of $41.5 \%$. Twenty-two examinees (47\%) scored less than $40 \%$ (Fig. 3 ) and 35 examinees $(74.5 \%)$ scored less than $50 \%$ (Fig. 4). The average score for each subject broken down into the following sub sections was; safety $(60.5 \%)$, medical terminology $(45 \%)$, basic principles of MRI (39.5\%), pulse sequences (37.5\%), image optimization (26.5\%), anatomy (43.5\%) and pathology (57\%) (Fig. 1). Twenty examinees (42.5\%) did not know all the safety contraindications to MRI (Fig. 7). Fifteen (38.5\%) of the 39 individuals who were asked to identify an unlabelled diagram of an X-ray tube were unable to do so (Fig. 5).

When comparing the results of UK (Sample A) and non- UK (Sample B) trained individuals, there was little difference in their average scores (UKZ43\%, non UKZ40\%). The average scores for each subject were similar except medical terminology, anatomy and pathology where the UK trained examinees scored more highly than the non-UK trained examinees (Fig. 2). Eight (33\%) of non-UK trained examinees and $4(27 \%)$ of UK trained examinees could not recognize an unlabelled diagram of an X-ray tube (Fig. 6). Thirteen (40\%) of non-UK trained examinees and $7(46 \%)$ of UK trained examinees did not know all the contraindications to MRI (Fig. 8).

Only some examinees in Sample A (23) were given a viva voce examination. This was mainly due to other commitments the examinees had as part of the induction process for the private healthcare provider employing them. This represents $74 \%$ of Sample A across a range of abilities based on the OSCE score. All 23 examinees were asked questions relating to the cervical spine X-ray photograph. Fourteen were also given the patient focused viva voce questions because they had scored less than $50 \%$ in the OSCE. Nine were also given the more advanced MRI questions because they had scored more than $50 \%$ in the OSCE. The viva voce examinations were not scored but responses were mapped against those thought acceptable by the assessors. Most examinees responded appropriately to questions posed on patient related matters although there were some notable exceptions which are discussed later. None of the examinees who were asked the more advanced MRI questions were able to answer more than two questions correctly. All examinees were asked to identify the examination being undertaken from a photograph of a patient having a lateral cervical spine X-ray study. Three of these examinees could not do so correctly and 7 examinees gave incorrect answers to questions posed about this photograph. Ideally all 47 examinees would have been assessed using the same questions and the same assessment tools (OSCE and viva voce). However for the reasons outlined above this was not possible and we recognise this as a limitation of this study.

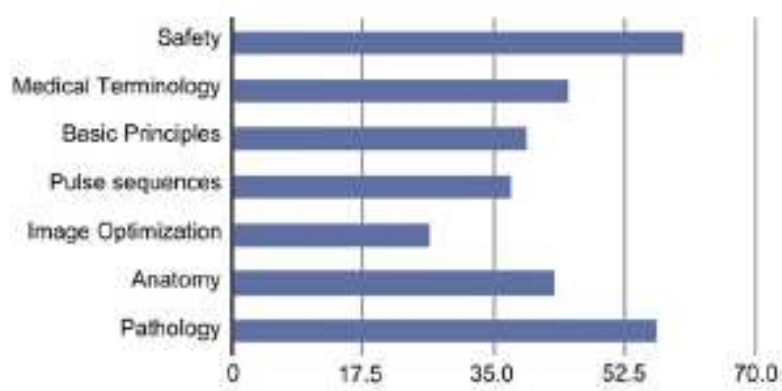

Figure 1 Average OSCE scores for each sub section. 


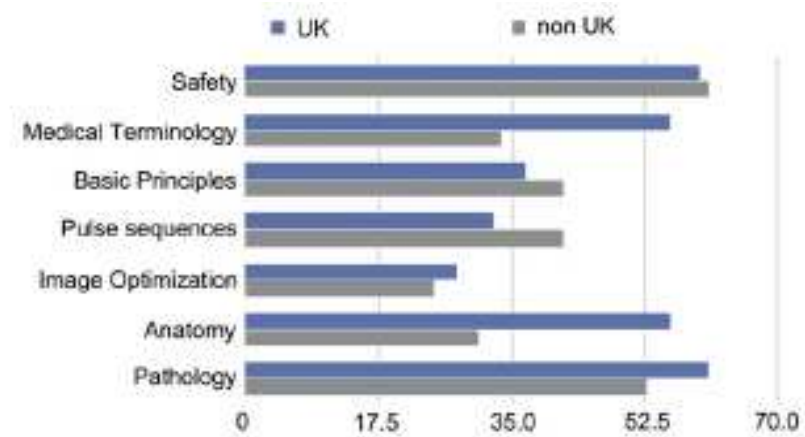

Figure 2 Average OSCE scores for each sub section; UK vs non-UK trained individuals.

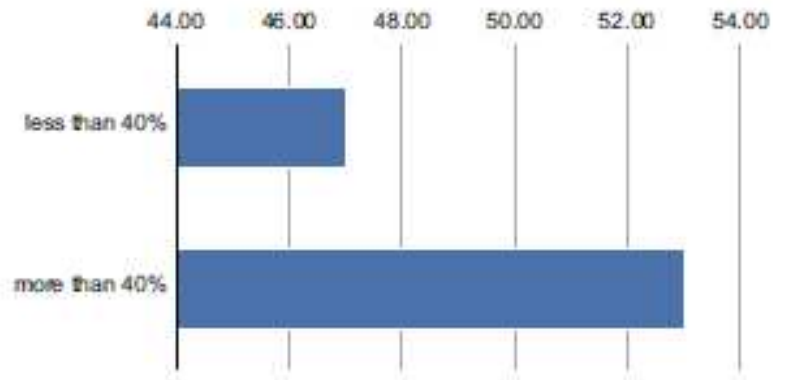

Figure $3 \%$ of Examinees scoring $40 \%$ or less on overall OSCE score.

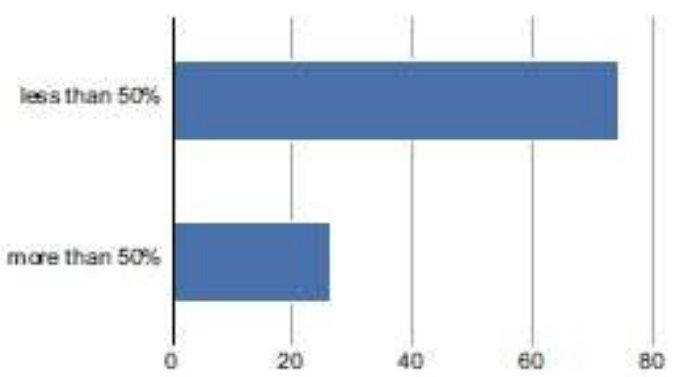

Figure $4 \%$ of Examinees scoring $50 \%$ or less on overall OSCE score.

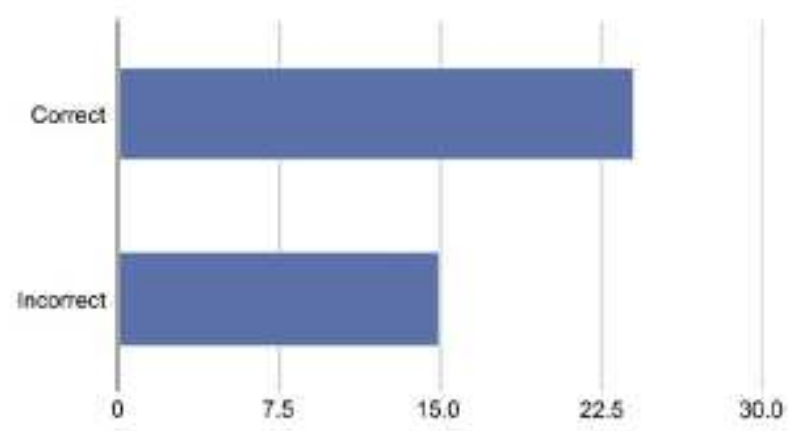

Figure 5 Examinees responses to identifying a diagram of an $X$-ray Tube. 


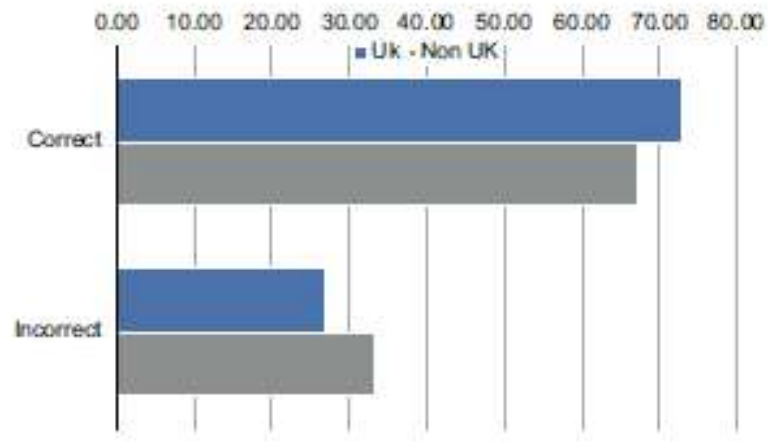

Figure $6 \%$ of UK vs non-UK responses to identify a diagram of an X-ray Tube.

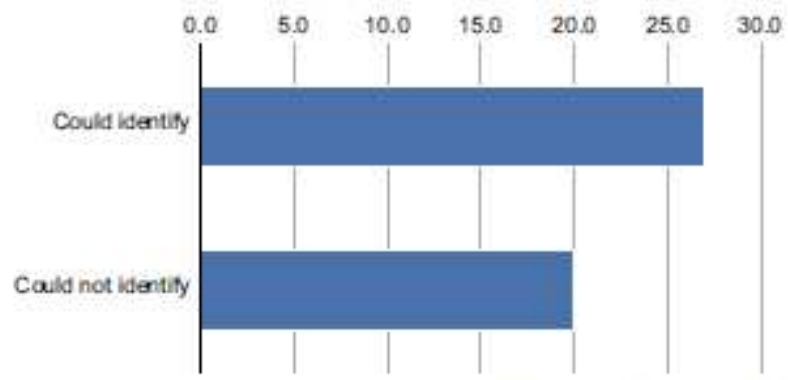

Figure 7 Number of examinees able to identify all 4 contraindications to MRI.

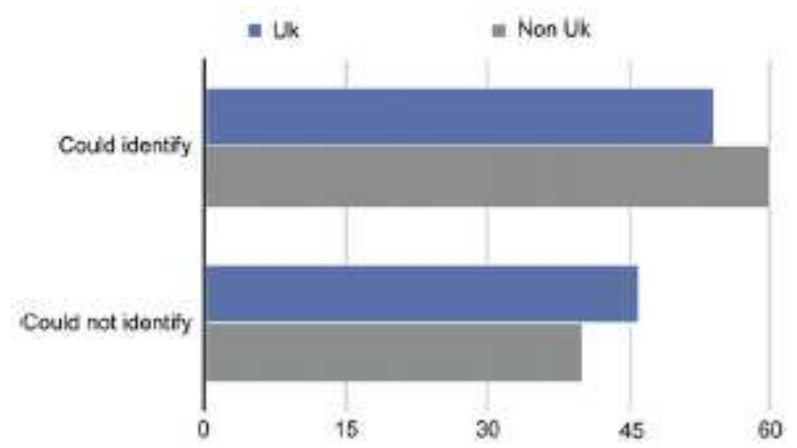

Figure 8 Number of examinees able to identify all 4 contraindications to MRI; UK vs non-UK trained individuals.

\section{Discussion}

As far as we know, this was the first objective assessment of MRI knowledge related to clinical practice in a group of radiographers. Taber [20] states that; "the purpose of assessment is to judge what learning has taken place". In the context of this research, assessment tools were implemented to test the learning that had been facilitated mainly by self learning, or by other practitioners and applications specialists. As previously outlined, the OSCE was chosen as the most appropriate method as it is highly viable and valid and it provides objective information about the clinical performance of individuals [19]. As there is no standard examination in MRI in the UK, there were no benchmarks to use when compiling the questions for the OSCE. The questions we used were largely based on the ARRT content specifications for their examination in MRI which we felt were representative as the range of knowledge tested was mainly at a basic level and related to practice [14].

The viva voce examination is a more subjective assessment tool, however it can be very effective in eliciting specific information about an individual's knowledge. We implemented this for certain examinees as part of a wider assessment process. Specifically it enabled us to further establish the radiographic credentials of most of the examinees in Sample A. Ideally all 
individuals in both sample populations would have received the viva voce examination. This was not possible due to limitations previously outlined. In addition, as the need for a general radiographic question was not identified in the pilot study and only became apparent in the first batch of examinees in Sample A, the X-ray tube question was only given to 39 examinees. If the pilot study had been carried out on the same population as Samples $A$ and $\mathrm{B}$, the need for an assessment on general radiographic knowledge would have been ascertained earlier. However this would have meant that the sample population would have answered the OSCE questions twice and would have affected the results. It was deemed important that the OSCE tested the entry level knowledge of each examinee without prior revision or knowledge of the questions. This is why the pilot study was carried on three examinees who were not part of the sample population. Two of the individuals in the pilot study were radiographers but, as they scored well, the need for a general radiographic question was not highlighted. Despite these limitations however we believe that we were able to assess the knowledge related to the safe use of an MRI scanner in a meaningful way. Although the sample size was relatively small when compared to the total number of practicing MRI radiographers, the examinees had a wide range of experience in MRI and had learnt MRI from a representative range of sources and in a variety of geographical locations.

In general the overall OSCE scores were disappointing. Although, a low score in technical subjects like basic principles and pulse sequences might be expected, it is worrying that overall nearly three quarters of the examinees scored less than $50 \%$ in the OSCE despite some having more than 10 years experience. Two of the examinees were application specialists and therefore responsible for the training of others. Although these two radiographers scored more highly than others, they were still unable to answer very basic questions correctly particularly relating to image optimisation, pulse sequences and safety. There was little difference in the overall scores between UK (Sample B) and non-UK trained radiographers (Sample $A$ ), suggesting that the provision of MRI training in the countries represented is similar. Perhaps not surprisingly, non-UK trained radiographers scored less than UK trained radiographers in medical terminology, however as all the non-UK radiographers tested were HPC registered, lack of knowledge in this subject must raise concerns about the ability of these practitioners to communicate effectively in the clinical setting.

Two areas of particular concern in the OSCE were MRI safety and image optimisation. Over $42 \%$ of the examinees did not know the four main contraindications to MRI and this was higher in UK trained radiographers (46\%) than non- UK radiographers $(40 \%)$. These results are very alarming as, if patients with these contraindications are exposed to the high magnetic field of an MRI scanner, serious injury and death can occur. Knowledge of these contraindications is essential and the fact that so many examinees could not identify them is unacceptable. Image optimisation was the most poorly scoring section with an average overall score of $26.5 \%$ and both UK and non-UK trained radiographers scoring poorly ( $28 \%$ and $25 \%$ respectively). None of the examinees scored more than $40 \%$ in this section, including those radiographers with more than 10 years experience. Image optimisation relates to artefact recognition and the manipulation of parameters to optimise image quality. This subject is very important to MRI radiographers as it concerns the production of the highest quality diagnostic images enabling effective interpretation and diagnosis. Radiographers are required to understand concepts such as signal to noise ratio, contrast to noise ratio, scan time and spatial resolution and have a thorough grasp of the wide variety of selectable parameters and how they "trade-off" against each other [23]. Image optimisation is uniquely a radiographer's responsibility, forms the bedrock of MRI radiographic practice and a lack of understanding of this subject can result in poor quality images, inefficient use of the scanner and misdiagnosis [22].

However the majority of radiographers we tested did not possess even basic knowledge of image optimisation. The reasons for this are probably due to a combination of factors. All MRI manufacturers use operator interfaces that can automate the scanning process. In its most basic form this automation involves pre-selected protocols that are initially set up by the manufacturers and their applications specialists. Radiographers still have to prescribe slices through the region of interest and initiate the scan process but all the parameters within the protocol are fixed. Although parameters can be changed, this is usually performed in centres 
where practitioners are knowledgeable and understand the consequences in terms of image quality and image contrast [24]. Recently the automated process has been extended by some manufacturers to include slice prescription and scan initiation so that radiographic input in terms of the technical process of the scan is limited to coil selection and patient set up [17]. Although the use of pre-defined protocols enables high patient throughput and image reproducibility, they tend to inhibit parameter modification and limit radiographic learning of image optimisation parameters. This means radiographers are not only largely ignorant of an important part of the scanning process, they are ill-equipped to recognize artefactual image appearances and react to unusual clinical situations appropriately. It is imperative that these skills are acquired and retained by all MRI radiographers as it is essential that they are able to adapt protocols when encountering unusual scanning scenarios or where the pathology encountered requires decisions to be made that will alter how the protocol is run. However in order to do this, radiographers must have an intuitive knowledge of image optimisation and our results show that this may not be the case.

Some of the examinees in Sample A were asked general radiographic questions to establish their radiographic credentials. As previously discussed, this was required as individuals in Sample A were required to perform general radiographic examinations on a rotational basis. Their academic radiographic qualifications had been assessed by the HPC as part of the registration process. As such we had assumed that the radiographic credentials of these individuals were sound and had not intended assessing this further. However the scores of some of the first 8 individuals assessed via the OSCE were very low which caused us concern. To determine the radiographic credentials of the rest of the examinees in Sample A, we included questions we considered anyone who had studied radiography and performed general radiographic examinations would have been able to answer correctly. We added an unlabelled diagram of an X-ray tube to the OSCE and a photograph of a patient having a lateral cervical spine $\mathrm{X}$-ray examination to the viva voce. The $\mathrm{X}$-ray tube question was also added to the OSCE given to Sample B. As previously discussed, although these individuals were not required to perform general radiographic procedures in addition to their MRI duties, they were all qualified radiographers and we wanted to see whether the accuracy of their responses to this question was similar to those in Sample A.

The X-ray tube diagram was added as this is an iconic image that all radiographers should learn as part of their entry level qualification in radiography. Thirty-nine examinees were asked to identify the diagram and to not assume that the answer was related to MRI. This instruction was given to remove bias in the examinees responses who may have answered the question incorrectly as a result of the MRI focus of the OSCE. Accepted answers were " $X$ ray tube" and "rotating anode tube". In total $38.5 \%$ of the examinees either could not identify the diagram at all or identified it incorrectly. Most incorrect responses involved the examinee saying this diagram was a component of an MRI scanner, even though it does not resemble any part of the MRI system. More UK trained than non-UK trained radiographers identified the diagram incorrectly (33\% and $27 \%$ respectively). It is difficult to understand why so many radiographers failed this question.

Twenty-three non-UK trained individuals (Sample A) were given a viva voce and three (13\%) of these could not correctly identify the lateral cervical spine study. Two individuals thought the patient was having a chest examination and one could not even identify the part of the body being imaged. Seven (30\%), which included three examinees who could not identify the photograph, could also not correctly name some of the distances highlighted in the photograph. In particular four examinees could not identify the air gap and did not know what this was for. One examinee identified the collimator as "where the exposure factors are selected" and two did not know the purpose of setting a Kvp. These results are worrying and may be due to some individuals falsely claiming they were radiographers. However given that all were HPC registered this seems unlikely. Certainly these results should concern those delivering degree or diploma Radiographic courses.

Very few radiographers in Sample A could answer the advanced MRI viva voce questions despite scoring highly in the OSCE. Only two questions were answered correctly by two of the nine examinees who were asked these questions. Although these questions were deliberately difficult to elicit advanced level knowledge, it is disappointing that more were not answered 
correctly given that several of the examinees had many years experience in MRI and two were applications specialists. Most individuals provided appropriate answers to the patient focused questions. However there were some notable exceptions. One radiographer with three years, experience said that they would carry on scanning a patient who had pressed the alarm bell until "the doctor told him to stop". This obviously has serious safety implications as the patient may have raised the alarm for many reasons including life threatening causes. In addition one examinee said they would sedate a patient who had been doubly incontinent in the scanner. Nine of the examinees from India and Sri Lanka said that their response to an aggressive patient in the waiting room would be to "move them up the queue and scan them as soon as possible". This perhaps reflects a different approach to aggressive patients in these countries as compared to the UK. These results suggest that some radiographers may need particular training in certain aspects of patient care. As UK trained radiographers were not given a viva voce examination we could not assess their response to these questions or compare them to non-UK trained individuals. We recognize that this is a flaw and recommend further study.

The primary aims of this study are to assess the knowledge of a group of MRI radiographers. The OSCE scores and MRI related questions in the viva voce were generally very poor and lower than expected. Our results show a lack of clinically related basic knowledge. In particular, an unacceptable number of examinees could not correctly identify the four main contraindications to MRI and none of the examinees scored more than $40 \%$ in image optimization e a subject inherent to best practice. Our results suggest that in-house training is lacking and a significant number of MRI radiographers are unfamiliar with key MRI safety concepts. As previously discussed, although there are some short courses available, most radiographers do not attend or only do so after practicing for many years, rather than at an early stage of their training. Even fewer radiographers go onto study post-graduate qualifications where their level of knowledge is formally assessed. The reasons for nonattendance are varied but include a lack of funding, staffing issues and a lack of motivation. When MRI radiographic posts are plentiful and there is no requirement to pass examinations to practice MRI, there is little motivation to study to a high level. However our results suggests that the training of MRI radiographers is lacking and fundamental changes need to be implemented.

In the Unites States the radiographic registering body, the ARRT, require all their registrants participate in continuing education as part of a certification process. This can take the form of acquiring credits over a period of time through a variety of educational activities. However in specialties such as MRI this must include a biennial examination and keeping a record of clinical experience [1]. Accredited affordable training programmes are available across the United States to prepare for the examination and these courses are taught using learning outcomes and a syllabus provided by the ARRT. The examination, which is in a timed multiple-choice computer generated format similar to an OSCE includes a wide range of theoretical and clinical MRI topics. Examinees must attain an overall score equivalent of $65 \mathrm{e} 70 \%$ to pass. Failure results in certification being withdrawn by the ARRT. In many States certification is mandatory for all MRI radiographers to practice [1].

In the past, educational providers in the UK have resisted the introduction of similar testing methods. Multiple-choice examinations are often thought to be too easy and encourage surface or rote learning rather than deep learning [5]. In addition this method of assessment is criticized as it may allow students to pass by simply guessing the answer [11]. Regulatory bodies such as the HPC have focused on continuing professional development (CPD) activities. Recently all registrants of the HPC are required to maintain CPD files which contain evidence of their educational activity. They submit this to the HPC if requested to do so along with a CPD profile where the radiographer explains how the evidence in their CPD file shows they have met the required standards [12]. Whilst this process motivates radiographers to undertake regular CPD activities, it is not certain whether it will directly and objectively assess factual learning. Will it, for example, be able to prove that an MRI radiographer is aware of the contraindications to MRI? In our view, attendance at an MRI safety lecture does not necessarily prove that the individual has learnt the contraindications to MRI. Even a statement by the radiographer in their CPD profile that they have learnt MRI safety cannot prove that this knowledge is in place e it can only be implied. Whilst CPD activities are valuable in 
encouraging radiographers to reflect on their learning, assessment of this type can only be valid if basic knowledge is already in place. Reflective writing and evidence gathering are subjective and its ability to test factual information is limited. An objective assessment method such as an OSCE, that asks a direct question and requires the radiographer to provide a direct answer, is a more meaningful assessment tool for this purpose [13].

Our results show that the majority of radiographers in our sample could not answer more than half the OSCE questions correctly and a significant and unacceptable number did not convince us they could practice MRI safely. Very few of the examinees had adequate knowledge of image quality issues and parameter manipulation. A few also raised concerns over their basic radiographic credentials. Professional bodies and radiographers themselves have a responsibility to assure the public and the wider medical community that radiographers have the knowledge and skills to practice safely and competently. Our results suggest that this cannot be assumed, despite the introduction of the CPD process.

We recommend establishing a standardized training programme with mandatory assessment of factual knowledge related to the safe practice of MRI. This would not replace what already exists but supplement it. Dialogue between educational professionals and MRI radiographers is necessary to establish the most appropriate learning outcomes and a syllabus designed to support these. Teaching strategies that encourage the integration of theory and practice must be implemented. The delivery of the syllabus should be monitored to ensure that teachers are appropriately qualified and that the syllabus is being taught to a high standard. In addition, valid and reliable assessment tools must be implemented that can be administered to large number of examinees. Results must include information on performance in each subject with strengths and weaknesses highlighted so that these can be communicated to the examinee and used for future CPD. These results must also be used to direct new teaching and learning and there should be a requirement to repeat this assessment on a regular basis. Where additional information is required, a face-to-face assessment such as a vive voce examination may be implemented.

Although a multiple-choice examinations and OSCE have disadvantages, these can be addressed by carefully selecting questions and implementing certain marking criteria. By setting appropriate questions, the level of difficulty can be increased to reflect the type of learning to be assessed. Questions that require comprehension and application skills can also be used to distinguish the surface from the deeper learner [11]. The problem of some students being able to pass by guessing the answer can be largely eliminated by using negative marking strategies, increasing the number of answer choices or by raising the pass mark to a level beyond which an examinee can pass by chance [15]. The lack of knowledge of image optimization, parameter manipulation and artefact recognition must be addressed within the curriculum and assessed in detail. Support from manufacturers in the development of operator interfaces that encourage learning in this regard rather than inhibit it, is vital. Manufacturers need to cooperate with educational professionals and clinical practitioners to ensure that radiographers participate in deep learning activities via their interaction with the scanner software. They must be able to solve problems, react to unusual clinical situations and possess intuitive knowledge of parameter manipulation and trade-offs. These are essential radiographic skills in MRI and therefore the user interface must encourage the application of these skills.

\section{Conclusion}

The main aim of this study was to assess a number of MRI radiographers' basic knowledge related to safe practice of MRI using an OSCE and therefore ascertain what MRI radiographers really know. Our results suggest that, despite the introduction of CPD, there is a significant lack of clinically related basic knowledge in people who claim to be experienced MRI users. We cannot therefore assume that all MRI radiographers are safe and competent to practice and, without some form of objective assessment of factual knowledge, we cannot know what radiographers have really learnt. This process is only meaningful if regular mandatory assessment is enforced. Whilst the OSCE method of assessment may not be ideal, some assessment of factual knowledge is better than no assessment at all. Although there are undoubtedly funding issues relating to this idea, we believe our results suggest that 
this is required to enable radiographers to fulfill their responsibility to maintain competence and prevent professional obsolescence.

\section{References}

[1] ARRT. Continuing education requirements for renewal of registration, http://www.arrt.org/education/cereq.pdf ; 2007 [accessed 26.02.08].

[2] Bannister S. Developing objectives and relating them to assessment faculty centre for teaching and e-learning, http://www.fctel.uncc.edu/pedagogy/assessment/DevelopingLearningOutcomes.html ; 2000 [accessed 16.11.07].

[3] Biggs J. Teaching for quality learning at university. Buckingham, UK: Open University Press; 2003.

[4] Burton S, Sudweeks R, Merrill P, Wood B. Multiple choice test e guidelines for a University faculty. Brigham Young University; 1991.

[5] Brown G. Assessment: a guide for lecturers learning and teaching support network (LTSN); 2001.

[6] College of Radiographers. Directory of pre-registration courses, http://www.sor.org/public/documentlibrary/sor directory pre registration courses.pdf 2007 [accessed 12.06.08].

[7] College of radiographers. Directory of post-graduate courses, http://www.sor.org/public/document-library/sor directory postgraduate courses.pdf 2007 [accessed 12.06.08].

[8] DeLisa JA. Certifying and measuring competency in the United States. Arch Phys Med Rehabil 2000;81:1236e41.

[9] Dunn L, Morgan C, O'Reilly M, Parry S. The student assessment handbook. Routledge; 2004.

[10] Hamadeh G, Lancaster CJ, Johnson AH. Introducing the objective Structured Clinical Examination to a family medicine residency program. Fam Med 1993;25(4):237e41.

[11] Higgins E, Tatham L. Exploring the potential of multiple-choice questions in assessment learning and teaching unit. Manchester Metropolitan University; 2003.

[12] HPC. Your guide to our standards for continuing professional development, http://www.hpc-uk.org/assets/documents/1000119FShort guide to CPD.pdf 2006 [accessed 27.03.08].

[13] Jain SS, Nadler S, Eyles MY, Kirshblum S, DeLisa JA, Smith A. Development of an objective structural clinical examination (OSCE) for physical medicine and rehabilitation residents. Am J Phys Med Rehabil 1997;76:102e6.

[14] Kaut C, Faulkner W. Review questions for MRI. Blackwell Science; 1995.

[15] McKenna C, Bull J. Designing effective objective questions: an introductory workshop, http://caacentre.lboro.ac.uk/dldocs/otghdout.pdf; 1999 [accessed 30/3/08].

[16] Nicol M, Freeth D. Assessing comprehensive nursing performance: the objective structural clinical assessment. Nurse Educ Today 1998;18:601e9.

[17] Philips Medical Systems. Smart scan, http://www.medical.philips.com/main/products/mri/technology/smartexam/index.html 2008 [accessed 26.03.08].

[18] Rust C. The impact of assessment on student learning. Active Learn High Educ 2002;3(2):145e8.

[19] Sloan DA, Donnelly MB, Scwartz RW, Strodel WE. The objective structured clinical examination. The gold standard for evaluating postgraduate clinical performance. Ann Surg 1995; 222(6):735e42.

[20] Taber K. Examining structure and context e questioning the nature and purpose of summative assessment. In: Seminar presentation to Cambridge International Examinations University of Cambridge Local Examinations Syndicate; 2003.

[21] White C. Assessing key skills by video or audio presentation, higher education academy, http://www.heacademy.ac.uk/assets/York/documents/resources/resourcedatabase/id 395 assessing key skills.pdf 2004 [accessed 28.11.07].

[22] Westbrook C. Handbook of MRI technique. 2nd ed. Blackwell Science; 1999.

[23] Westbrook C, Kaut-Roth C, Talbot J. MRI in practice. 3rd ed. Blackwell Science; 2005.

[24] Westbrook C. Image Optimization. Invited lecture at the Society of Magnetic Resonance Imaging conference, Melbourne Australia; 2007. 60 C. Westbrook, J. Talbot 